\title{
Lemaître Transformations of the Interior Schwarzschild Metric
}

\section{Rainer Burghardt}

A-2061 Obritz 246, Obritz, Austria

Email:arg@aon.at

How to cite this paper: Burghardt, R. (2021) Lemaitre Transformations of the Interior Schwarzschild Metric. Journal of Modern Physics, 12, 99-104.

https://doi.org/10.4236/jmp.2021.122010

Received: December 14, 2020

Accepted: January 22, 2021

Published: January 25, 2021

Copyright $\odot 2021$ by author(s) and Scientific Research Publishing Inc. This work is licensed under the Creative Commons Attribution International License (CC BY 4.0).

http://creativecommons.org/licenses/by/4.0/

\begin{abstract}
A Lemaitre transformation is set up for the free fall in the interior of a stellar object using the frame of the interior Schwarzschild solution. The metric is calculated in comoving coordinates and field strengths are derived for this metric.
\end{abstract}

\section{Keywords}

Interior Schwarzschild Metric, Lemaitre Transformations, Freely Falling Systems

\section{Introduction}

In this short note, we supplement investigations of an earlier paper [1] on free fall inside a non-rotating stellar object, which is described by the interior Schwarzschild solution. In the above mentioned paper, we had set up a Lorentz transformation that describes the physics of an observer who is moving in free fall through a tube bored through the center of the star. We assign a matrix to this Lorentz transformation that describes a coordinate transformation with which the metric of the interior Schwarzschild solution can be brought into a form that corresponds to Lemaitre's relation for free fall in the exterior Schwarzschild field.

We also discuss the form parameter of the metrics in Schwarzschild static coordinates and Lemaître coordinates, and derive the field quantities from tetrads corresponding to the metric in Lemaitre form.

\section{Free Fall Inside a Star}

We started with the metric of the Schwarzschild interior solution

$$
\mathrm{d} s^{2}=\frac{1}{1-\frac{r^{2}}{R^{2}}} \mathrm{~d} r^{2}+r^{2} \mathrm{~d} \vartheta^{2}+r^{2} \sin ^{2} \vartheta \mathrm{d} \varphi^{2}-\frac{1}{4}\left[3 \cos \eta_{g}-\cos \eta\right]^{2} \mathrm{~d} t^{2}
$$


in the form given by Flamm [2]. The lapse function of the metric is

$$
a_{T}=\frac{1}{2}\left[3 \cos \eta_{g}-\cos \eta\right] .
$$

Here, $r$ is the radial coordinate, $\eta$ is the polar angle of a cap of a sphere with radius $R$, representing geometrically the interior Schwarzschild solution. $\eta_{g}$ is the polar angle at the boundary surface separating the interior solution from the exterior solution.

From

$$
v_{T}^{2}+a_{T}^{2}=1
$$

we deduced the velocity of free fall

$$
v_{T}=-\sqrt{1-\frac{1}{4}\left(3 \cos \eta_{g}-\cos \eta\right)^{2}}
$$

and with $\alpha_{T}=a_{T}^{-1}$ the assigned Lorentz factor

$$
\alpha_{T}=\frac{2}{3 \cos \eta_{g}-\cos \eta} .
$$

Finally, we obtain the Lorentz transformation

$$
L_{1^{\prime}}^{1}=\alpha_{T}, \quad L_{4^{\prime}}^{1}=-i \alpha_{T} v_{T}, \quad L_{1^{\prime}}^{4}=i \alpha_{T} v_{T}, \quad L_{4^{\prime}}^{4}=\alpha_{T}
$$

operating in the $[1,4]$-slice of the model. We use the original Minkowski notation with

$$
x^{4}=i(c) t .
$$

At the boundary one has $\eta=\eta_{g}$ and thus

$$
v_{T}^{g}=-\sin \eta_{g}, \quad \alpha_{T}^{g}=\frac{1}{\cos \eta_{g}}=\frac{1}{\sqrt{1-v_{T}^{g 2}}} .
$$

The cap of the sphere provides the relations

$$
r=R \sin \eta, \quad \cos \eta=\sqrt{1-\frac{r^{2}}{R^{2}}} .
$$

From Flamm's paper [2] we read $\rho=2 R$, where

$$
\rho=\sqrt{\frac{2 r^{3}}{M}}, \quad \mathrm{R}=r \sqrt{\frac{r}{2 M}}
$$

is the curvature radius of the Schwarzschild parabola and the radius of a cap of a sphere. Thus, we have $\sin \eta=\sqrt{2 M / r}$. Finally, we obtain at the boundary

$$
v_{T}^{g}=-\sqrt{\frac{2 M}{r_{g}}}, \quad \alpha_{T}^{g}=\frac{1}{\sqrt{1-\frac{2 M}{r_{g}}}}
$$

the values for an observer freely falling in the exterior field having reached the boundary.

Thus, using for the velocity the expression 


$$
v_{T}(r)=-\sqrt{1-\frac{1}{4}\left(3 \sqrt{1-\frac{2 M}{r_{g}}}-\sqrt{1-\frac{2 M r^{2}}{r_{g}^{3}}}\right)^{2}}
$$

we are prepared for a drawing. We note that at the center of the star $(r=0)$ the velocity of the freely falling observer would reach the velocity of light for $r_{g}^{\min }=2.25 M$. We call this the inner horizon of the Schwarzschild model. This is the minimal extension of a star of mass $M$ in geometrical units. We extended the problem for an object freely falling from an arbitrary position outside of the star [3] [4]. This is depicted in Figure 1.

The surface of the stellar object is indicated by the dashed lines. One can recognize the smooth transition of the velocities from the exterior region to the interior region. The pressure of the star would be infinitely high at the inner horizon $r_{\min }=2.25 M$. Hence, it is evident that one has to accept that $r_{g}>r_{\min }$. Since $r_{\min }>2 M$ a formation of a black hole is not possible with the frame of the complete Schwarzschild model. In [5] we have shown that a collapsing star can reach the inner horizon only asymptotically, i.e., after an infinitely long time.

\section{The Lemaître Transformation}

The fact that the free fall velocity of an object in the exterior field can be easily prolongated into the interior raises the question whether a coordinate transformation changes the interior metric into a form which has the structure of the exterior Lemaitre metric can be found. Since the problem can be reduced to the $[1,4]$-slice of the model, it is sufficient to read from the interior metric (2.1) the bein-vectors

$$
\stackrel{1}{e_{1}}=\alpha_{I}=\frac{1}{\sqrt{1-\frac{r^{2}}{R^{2}}}}=\frac{1}{\cos \eta}, \stackrel{4}{e_{4}}=a_{T}, \underset{1}{e}=a_{I}=\sqrt{1-\frac{r^{2}}{R^{2}}}=\cos \eta, \underset{4}{e^{4}}=\alpha_{T} .
$$

With the Lorentz transformation (2.6), we are able to transform the beine into the freely falling system with ${\stackrel{m}{e^{\prime}}}_{i}=L_{m}^{m^{\prime}} \stackrel{m}{e}_{i}$, still using the static coordinate system

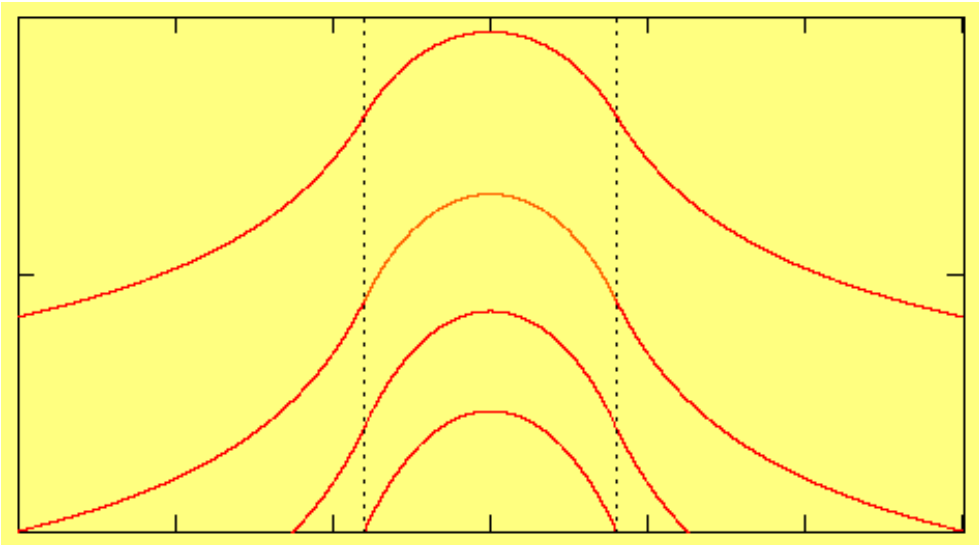

Figure 1. Free fall through the interior of a stellar object. 
(i). We try to diagonalize the bein system with an additional coordinate transformation:

$$
\begin{aligned}
& {\stackrel{m^{\prime}}{e^{\prime}}}_{i^{\prime}}=L_{m}^{m^{\prime}} \stackrel{m}{e}_{i} \Lambda_{i^{\prime}}^{i} \\
& \Lambda_{1^{\prime}}^{1}=-\alpha_{T} v_{T} a_{I}, \quad \Lambda_{4^{\prime}}^{1}=-i \alpha_{T} v_{T} a_{I}, \quad \Lambda_{1^{\prime}}^{4}=-i \alpha_{T}^{2} v_{T}^{2}, \quad \Lambda_{1^{\prime}}^{4}=\alpha_{T}^{2} \\
& \Lambda_{1}^{1^{\prime}}=-\frac{\alpha_{T} \alpha_{I}}{v_{T}}, \quad \Lambda_{4}^{1^{\prime}}=-i, \quad \Lambda_{1}^{4^{\prime}}=-i \alpha_{T} v_{T} a_{I}, \quad \Lambda_{1}^{4^{\prime}}=1 .
\end{aligned}
$$

Indeed, we obtain

$$
{\stackrel{1}{e^{\prime}}}_{1^{\prime}}^{\prime \prime}=-v_{T}, \quad \stackrel{4}{e}_{4^{\prime}}^{\prime}=1, \quad e_{1^{\prime}}^{1^{\prime}}=-\frac{1}{v_{T}}, \quad e_{4^{\prime}}^{4^{\prime}}=1,
$$

recalling that $v_{T}$ is pointing inwards and thus is a negative quantity.

It is easy to show that

$$
\Lambda_{[i \mid k]}^{j^{\prime}}=0, \quad \Lambda_{\left[i^{\prime} \mid k\right]}^{j}=0 \Rightarrow \Lambda_{i}^{j^{\prime}}=x^{j^{\prime}}, \quad \Lambda_{i^{\prime}}^{j}=x_{\mid i^{\prime}}^{j},
$$

proving that the new Lemaitre coordinates are holonomic. Restricting ourselves to the $[1,4]$-slice of the model, we obtain for the line element

$$
\mathrm{d} s^{2}=v_{T}^{2} \mathrm{~d} r^{\prime 2}-\mathrm{d} t^{\prime 2}
$$

using comoving coordinates $\left\{r^{\prime}, t^{\prime}\right\}$. Evidently, taking $v_{T}$ at the boundary the line element (3.6) coincides with the well-known Lemaitre line element of the exterior solution. Comparing it with the line element in the non-comoving coordinates in the canonical form given by (2.1), we recognize that the form parameter is $k=1$ for the static line element and $k=0$ for the freely falling coordinate system. We recall that $k$ is misleadingly called curvature parameter by cosmologists and $k=0$ is believed to describe a globally flat geometry. Once more, we convince ourselves that $k=0$ indicates a locally flat system, i.e., a system being in free fall. More on this topic one could be found in our papers [6] [7].

In addition, we read from (3.6) that the time $t^{\prime}$ is the same for all observers at any point during the free fall. The coordinate time coincides with the proper time and is the universal time for all freely falling observers.

Since the lapse function is $g_{4^{\prime} 4^{\prime}}=1$, one cannot draw gravitational forces from the metric of the freely falling system. This is just what we expect from Einstein's elevator principle [6]. We calculate the Ricci-rotation coefficients from the comoving bein system (3.4). At first we obtain

$$
' U_{4^{\prime}}=A_{1^{\prime} 4^{\prime}}^{1^{\prime}}=-e_{1^{\prime}}^{1^{\prime}} e^{1^{\prime} \mid 4^{\prime}}=\frac{1}{v_{T}} v_{T \mid 4^{\prime}}=\frac{1}{v_{T}}\left(-i \alpha_{T} v_{T}\right) v_{T \mid 1}=-i \alpha_{T} v_{T \mid 1} .
$$

For further processing one derives from Equation (2.7) the relations $\mathrm{d} r=\mathrm{R} \cos \eta \mathrm{d} \eta, \mathrm{d} x^{1}=(1 / \cos \eta) \mathrm{d} r=R \mathrm{~d} \eta$ and finally

$$
\eta_{\mid 1}=\frac{1}{R} \text {. }
$$

Differentiating (2.4) one arrives at 


$$
v_{T \mid 1}=-\frac{1}{2} \frac{a_{T}}{v_{T}} \frac{1}{\mathrm{R}} \sin \eta
$$

and lastly we get with $\alpha_{T}=1 / a_{T}$ the tidal force

$$
\text { ' } U_{m^{\prime}}=\left\{0,0,0, i \frac{\sin \eta}{v_{T}} \frac{1}{2 R}\right\} .
$$

Once again using (2.4) one obtains with $v_{T}^{g}=-\sin \eta_{g}$ and $\rho_{g}=2 \mathrm{R}$

$$
{ }^{\prime} U_{m^{\prime}}^{g}=\left\{0,0,0,-\frac{i}{\rho_{g}}\right\},
$$

the corresponding expression for the free fall in the exterior field at the boundary. We note that we could also apply the inhomogeneous transformation law of the Ricci-rotation coefficients to transform the force of gravity [1] into (3.7). For $\eta=0$, i.e., at the center of the star one has ${ }^{\prime} U_{m^{\prime}}=0$.

This investigation is not merely an academic exercise. Benish [8] has treated the question whether the theoretically discussed process of free fall in the interior could be supported by an experiment. He proposed a torsion balance, similar to the one used in the Cavendish experiment. Two massive spheres can move through greater spheres on approximately radial orbits and thus could perform an oscillatory motion. The velocity of this motion could be detected on the axis of the suspension by optical devices. The design proposed by Benish is shown in Figure 2.

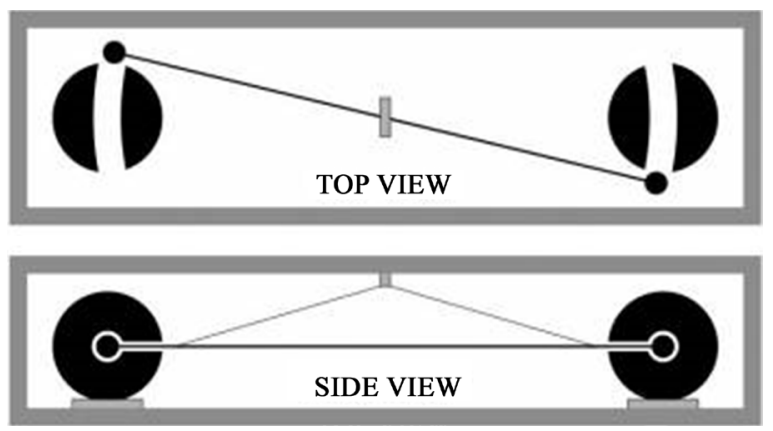

Figure 2. Experiment proposed by Benish.

\section{Conclusion}

We investigated the free fall through a non-rotating stellar object. We found that the velocity of free fall in the exterior Schwarzschild field is smoothly prolongated into the interior. According to Einstein's elevator principle no gravitational forces can be detected by a freely falling observer, but tidal forces act on him. We found a transformation from non-comoving coordinates to comoving coordinates and a metric in Lemaitre form. The form parameters of the metrics change from $k=1$ to $k=0$ by this transformation, demonstrating that $k=0$ is an indicator for free fall.

\section{Conflicts of Interest}

The author declares no conflicts of interest regarding the publication of this paper. 


\section{References}

[1] Burghardt, R. (2009) Interior Schwarzschild Solution and Free Fall. Austrian Reports on Gravitation. http://www.arg.or.at/Wpdf/WIff.pdf

[2] Flamm, L. (1916) Physikalische Zeitschrift, 17, 488-454.

[3] Burghardt, R. (2020) Spacetime Curvature. 1-688. http://arg.or.at/EMono.htm

[4] Burghardt, R. (2020) Raumkrümmung. 1-712. http://arg.or.at/Mono.htm

[5] Burghardt, R. (2015) Journal of Modern Physics, 6, 1895-1907. https://doi.org/10.4236/jmp.2015.613195

[6] Burghardt, R. (2016) Journal of Modern Physics, 7, 2347-2356. http://dx.doi.org/10.4236/jmp.2016.716203

[7] Burghardt, R. (2019) Journal of Modern Physics, 10, 1439-1453. https://doi.org/10.4236/jmp.2019.1012096.

[8] Benish, R.J. (2010) Interior Solution Gravity Experiment. https://de.scribd.com/document/9689809/Interior-Solution-Gravity-Experiment 